\title{
A super massive black hole binary in 3C 66B: Future observational perspectives
}

\author{
F. De Paolis, G. Ingrosso, and A. A. Nucita \\ Dipartimento di Fisica, Università di Lecce, and INFN, Sezione di Lecce, Via Arnesano, CP 193, 73100 Lecce, Italy \\ e-mail: depaolis@le.infn.it
}

Received 23 February 2004 / Accepted 27 June 2004

\begin{abstract}
Supermassive black hole binaries (SMBHBs) may exist in the centers of galaxies and active galactic nuclei (AGN) and are expected to be fairly common in the Universe as a consequence of merging processes between galaxies. The existence of SMBHBs can be probed by looking for double nuclei in galaxy centers or, more easily, detecting periodic behavior in the observed radio light curves. In a recent paper, Sudou et al. (2003, Science, 300, 1263) announced the first direct observation of an SMBHB. Using VLBI observations they found that the unresolved radio core of the radio galaxy 3C 66B shows a well defined elliptical motion with a period of $1.05 \pm 0.03 \mathrm{yr}$, implying the presence of a couple of massive black holes in the center of the galaxy. In the present paper we study the astrophysical implications of the existence of such an SMBHB in 3C 66B. In particular we focus on the information that can be obtained from detecting a signal periodicity either in the X-ray and/or $\gamma$-ray light curves as a consequence of the motion of the black holes. These observations could be used to extract further information on the physical parameters of the SMBHB and partially solve the system parameter degeneracy. The detection of the gravitational wave spectrum emitted by such system may be used to completely determine the physical parameters of the binary system.
\end{abstract}

Key words. gravitation - black hole physics - X-ray: general

\section{Introduction}

It is now well established that galaxies generally contain massive black holes in their nuclei (Rees 1984; Kormendy \& Richstone 1995; Richstone et al. 1998) and since merging events are expected to be rather common (see, e.g., White 1997) supermassive black hole binaries (hereinafter SMBHBs) may be found frequently in galactic cores (Begelman et al. 1980).

Up to now, several astrophysical phenomena have been attributed to SMBHBs. In fact, their presence influences the stars in the galaxy center regions whose mass density profile, as observed by numerical simulations (Nakano \& Makino 1991), may show a $\rho \propto r^{-1 / 2}$ profile typical of giant galaxies that have experienced many merging processes in their lifetime.

Moreover, if the black holes are rotating and have misaligned spins, plasma beams (aligned with the black hole axis) precess with period of $10^{3}-10^{4} \mathrm{yr}$ and curved jets should be observed. This phenomenon happens in double-double radio galaxies (Schoenmakers 2000) and in both S and X-shaped galaxies (Leahy \& Williams 1984; Parma et al. 1985; Wang et al. 2003).

Periodic outburst activity, as for example in the quasar OJ 287 (see, e.g., Sillanpää et al. 1998; Lehto \& Valtonen 1996) and periodic behavior in the radio, optical, $\mathrm{X}$-ray and $\gamma$-ray light curves of many AGNs are also possibly related to the presence of a central massive binary black hole creating a jet either aligned along the line of sight or interacting with an accretion disk (Yu 2002).

Up to now, the search for X-ray and/or $\gamma$-ray variability was considered as a method to probe the existence of an SMBHB in the center of a galaxy. For example, at least three Mkn objects (Mkn 501, Mkn 421 and Mkn 766) reveal periodic behavior in the observed signal so that in recent studies they have been considered as possibly containing SMBHBs (see Rieger \& Mannheim 2000; De Paolis et al. 2002, 2003).

However, the best tracer of the existence of SMBHB in a galaxy core remains the detection of the Keplerian orbital motion of some emission component close to the two black holes and, eventually, the observation of eclipse episodes as in the case of the AGN OJ 287 (Sadun et al. 1994; Lehto \& Valtonen 1996; Pietila 1998).

Unfortunately, a technical problem is that the typical black hole separation $\left(\sim 10^{16}-10^{17} \mathrm{~cm}\right)$, for a host galaxy located at the distance of $\simeq 100 \mathrm{Mpc}$, corresponds to a separation of $\sim 10-100 \mu \mathrm{arcsec}$ and only recently has the required accuracy in position measurements become available by the phasereferencing very long baseline interferometry technique at radio frequencies.

In a recent paper Sudou et al. (2003), using the VLBI facility, announced the first detection of a well defined Keplerian orbital motion of the radio emission component in a pair of 
well separated radio sources, $3 \mathrm{C} 66 \mathrm{~B}$ and $3 \mathrm{C} 66 \mathrm{~A}$. The natural explanation of this observation is that the radio galaxy $3 \mathrm{C} 66 \mathrm{~B}$ hosts an SMBHB at its center.

On the assumption that the SMBHB orbit is circular, Sudou et al. (2003) have estimated, by a best fitting procedure, the physical parameters of the system and found a mass density value of $2 \times 10^{15} M_{\odot} \mathrm{pc}^{-3}$, larger than of the massive object in NGC 4258 and SgrA*.

However, radio observations do not make it possible to determine all the binary system parameters since the results depend on the mass ratio $q$ between the two components, on the eccentricity of the system, and on the semi-major axis.

The aim of this paper is to present a complete analysis of the SMBHB in question by relaxing the assumption of a circular orbit ${ }^{1}$ and indicating some direction for further observations which may solve the parameter degeneracy for the system in $3 \mathrm{C} 66 \mathrm{~B}$.

In fact, a galaxy nucleus hosting an SMBHB of which one of the components is emitting a jet with Lorentz factor $\gamma_{\mathrm{L}}$ towards the observer may show periodic light curves both at X-ray and $\gamma$-ray wavelengths (see Rieger \& Mannheim 2000; De Paolis et al. 2002).

Thus, assuming that the radio jet in $3 \mathrm{C} 66 \mathrm{~B}$ is emitted by the less massive black hole (with mass $m$ ) which is moving in an elliptical orbit (with semi-major axis $a$ given by the VLBI radio observations, and generic eccentricity $e$ ) around its companion (having mass $M$ ), we study both the expected X-ray and $\gamma$-ray signal periodicity $P_{\text {obs }}$ and the flux ratio $f$ between maximum and minimum signal as a function of the jet Lorentz factor $\gamma_{\mathrm{L}}$ and of the power law spectral index $\alpha$ of the photon flux.

We show how the measurements of $f$ and $P_{\text {obs }}$ may be used to extract further information about the physical parameters of the SMBHB system hosted by the radio galaxy $3 \mathrm{C} 66 \mathrm{~B}$.

We also consider the gravitational wave spectrum and amplitude emitted by the SMBHB as a function of the orbital parameters of the system and compare the expected signal with the capabilities of the next generation space-based interferometers LISA and ASTROD.

The paper is structured as follows: in Sect. 2 we review the main properties of the radio galaxy $3 \mathrm{C} 66 \mathrm{~B}$ and in particular the observed parameters of the SMBHB at its center. In Sect. 3 we discuss the expected periodicity of the light curves in the X-ray and/or $\gamma$-ray band and show how the flux ratio $f$ depends on the system parameters. We show how one can use the expected periodicity $P_{\mathrm{obs}}$ and $f$ to constrain the physical parameters of the binary system. We have performed this analysis by assuming that the jet inclination angle to the line of sight is $i \simeq 1 / \gamma_{\mathrm{L}}$ as expected for typical blazars. In addition, we have considered also the more realistic case in which $i$ may be estimated by radio observations. However, in both cases the SMBHB parameters may be completely determined only if the spectrum of the gravitational waves emitted by the system is detected. Finally, our conclusions are given in Sect. 4.

\footnotetext{
${ }^{1}$ It is expected in fact that if SMBHBs come from merging events between galaxies they could be in elliptical orbits with eccentricities up to $0.8-0.9$ (Fitchett 1987).
}

Table 1. Parameters of the fitted elliptical motion of the radio core in $3 \mathrm{C} 66 \mathrm{~B}$ monitored in the two radio bands 2.3 and $8.4 \mathrm{GHz}$. Data are taken from Sudou et al. (2003).

\begin{tabular}{ccc}
\hline \hline Radio bands & $2.3 \mathrm{GHZ}$ & $8.4 \mathrm{GHZ}$ \\
\hline Semi-major axis $(\mu \operatorname{arcsec})$ & $243 \pm 30$ & $45 \pm 4$ \\
Axial ratio & $0.31 \pm 0.17$ & $0.24 \pm 0.14$ \\
Period $(\mathrm{yr})$ & $1.10 \pm 0.06$ & $1.02 \pm 0.04$ \\
\hline
\end{tabular}

\section{An SMBHB in the radio galaxy $3 \mathrm{C} 66 \mathrm{~B}$}

Using the very-long-baseline-interferometer, Sudou et al. (2003) recently announced the first direct evidence of the existence of an SMBHB in a distant galaxy.

They looked for the Keplerian orbital motion of a radio emission component in a pair of well separated $(\simeq 6$ arcmin) radio sources, $3 \mathrm{C} 66 \mathrm{~B}$ and $3 \mathrm{C} 66 \mathrm{~A} ; 3 \mathrm{C} 66 \mathrm{~B}$ is a radio galaxy at redshift $z=0.0215$ (Matthews et al. 1964) whereas 3C 66A is a more distant BL Lac object $(z=0.44$, Miller 1978) which was used as the stationary position reference to $3 \mathrm{C} 66 \mathrm{~B}$. In fact, observing both objects at 2.3 and $8.4 \mathrm{GHz}$ (Sudou et al. 2003) the radio core position of $3 \mathrm{C} 66 \mathrm{~B}$ at each epoch was measured with respect to $3 \mathrm{C} 66 \mathrm{~A}$. This revealed a time variation of the $3 \mathrm{C} 66 \mathrm{~B}$ core position which is well fitted by an elliptical motion with an averaged period estimated to be $1.05 \pm 0.03 \mathrm{yr}$ (see Table 1; and Sudou et al. 2003, for more details).

Since the radio core is located at the root of the jet where the optical depth for synchrotron self-absorption is of the order of unity (Lobanov 1998), it is expected that the $2.3 \mathrm{GHz}$ core is located at a greater distance from the central engine than the $8.4 \mathrm{GHz}$ core. Thus, the observation that the $2.3 \mathrm{GHz}$ semi-major axis $(243 \pm 30 \mu \mathrm{arcsec})$ is larger than the $8.4 \mathrm{GHz}$ one $(45 \pm 4 \mu \mathrm{arcsec})$ is a proof that the jet in $3 \mathrm{C} 66 \mathrm{~B}$ also suffers a precessional motion. According to Sudou et al. (2003), the most plausible explanation for this result is that while the $2.3 \mathrm{GHz}$ core motion maps the jet precession, the elliptic motion observed in the $8.4 \mathrm{GHz}$ band well describes the orbital motion of an SMBHB in 3C 66B with a Keplerian period $P_{\mathrm{K}}=1.05 \pm 0.03 \mathrm{yr}$.

Since, as stated above, observations at $8.4 \mathrm{GHz}$ are believed to reflect the radio core motion of the SMBHB more closely than those at $2.3 \mathrm{GHz}$, assuming that the jet is emanating from the less massive black hole (of mass $m<M$ ), Sudou et al. (2003) estimated the semi-major axis $a$ of the fitted orbit to be the upper limit of the orbital radius of the SMBHB, i.e.

$a_{\max } \simeq 5.4 \times 10^{16}(1+q) \mathrm{cm}$,

where $q=m / M$ is the mass ratio between the two binary components. With this assumption, and from the definition of the Keplerian period

$P_{\mathrm{K}}=2 \pi \sqrt{\frac{r^{3}}{G(M+m)}}$,

the following relation holds

$M \simeq 4.6 \times 10^{10}(1+q)^{2} M_{\odot}$, 


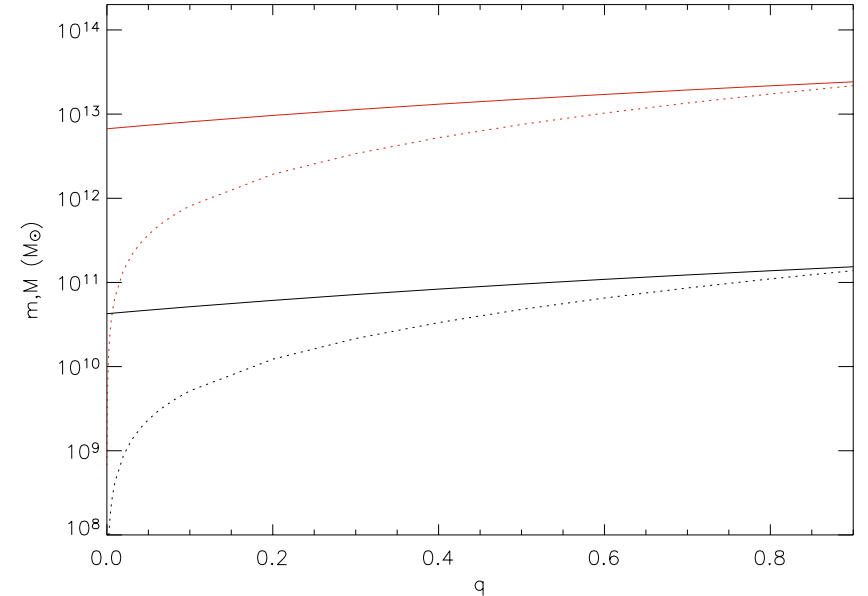

Fig. 1. The masses of the two black holes $m$ (dashed line) and $M$ (solid line) are given as a function of the mass ratio $q$. Red lines correspond to the orbital maximum semi-major axis $a \simeq 5.4 \times 10^{16}(1+q) \mathrm{cm}$ at $8.4 \mathrm{GHz}$ while black lines correspond to $a \simeq 2.9 \times 10^{17}(1+q) \mathrm{cm}$ at $2.3 \mathrm{GHz}$. (This figure is available in color in electronic form at www. edpsciences.org)

which makes it possible to estimate the masses of the two components once the mass ratio $q$ is given. In Fig. 1 the two black hole masses $m$ and $M$ of the black holes are shown as a function of the mass ratio $q$.

As is clear from Table 1, the estimated orbit semi-major axis $a$ is different at different wavelengths. In fact, it is $a_{\max } \simeq$ $5.4 \times 10^{16}(1+q) \mathrm{cm}$ at $8.4 \mathrm{GHz}$ and increases by a factor $\eta \simeq$ 5.4 at $2.3 \mathrm{GHz}$ becoming $a_{\max } \simeq 2.9 \times 10^{17}(1+q) \mathrm{cm}$. Thus, the mass $M$ in the previous equation is increased by a factor $\eta^{3}$ (as is also clearly stated in Sudou et al. 2003) if the semi-major axis is taken to be given by the observations at $2.3 \mathrm{GHz}$.

Here we would like to emphasize that observations at higher frequencies should allow to map more accurately the core motion in $3 \mathrm{C} 66 \mathrm{~B}$ giving a better estimate of the orbit semi-major axis which is expected to be smaller than $a_{\max } \simeq$ $5.4 \times 10^{16}(1+q) \mathrm{cm}$.

Sudou et al. (2003) assumed that the binary system is characterized by a circular orbit (i.e. $e=0$ ). However, if binary black holes originated from galactic mergers, they could be in eccentric orbits, and eccentricity values up to $0.8-0.9$ are not necessarily extreme (Fitchett 1987). Of course, due to gravitational wave emission, orbits tend to circularize but this happens within a time-scale of the same order of magnitude as the merging time-scale (Peters 1964; Fitchett 1987). Therefore, if an SMBHB is found at the center of a galaxy, it may happen that the components are still in eccentric orbits. Consequently, the axial ratio quoted in Table 1 gives an upper limit to the true orbital eccentricity $e$ since the binary orbit is seen projected on the plane of the sky. Thus, the possible SMBHB in 3C 66B may be characterized by an orbit with with an eccentricity up to $e_{\max } \simeq 0.96$. Relaxing the assumption of circular orbits will produce major changes in the estimation of both the gravitational wave amplitude and the coalescing time-scale of the SMBHB possibly hosted by $3 \mathrm{C} 66 \mathrm{~B}$.

\section{Signal periodicity in the X-ray and $\gamma$-ray light curves and the determination of the maximum-to-minimum flux ratio}

Periodic outburst activities and periodic behavior in the radio, optical, X-ray and $\gamma$-ray light curves of many AGNs are believed to be related to the presence of an SMBHB in the center of the host galaxy (Yu 2002).

On this basis, Rieger \& Mannheim (2000) (but see also De Paolis et al. 2002, for a generalization to orbits with generic eccentricity values) have recently proposed a method to determine the orbital parameters of the binary system from the observed quantities, i.e. the signal periodicity $P_{\text {obs }}$, the flux ratio $f$ between maximum and minimum signal and the power law spectral index $\alpha$ of the photon flux.

According to these models, the periodicity in the flaring state is assumed to be the consequence of the orbital motion of a relativistic jet in the binary black hole. Therefore, the observed signal periodicity has a geometrical origin due to Doppler-shifted modulation.

As a consequence of the binary orbital revolution around the center of mass, the expected signal period $P_{\mathrm{obs}}$ is related to the Keplerian period $P_{\mathrm{K}}$ by (Rieger \& Mannheim 2000)

$P_{\mathrm{obs}}=(1+z)\left(1-\frac{v_{z}}{c} \cos i\right) P_{\mathrm{K}}$

where, according to the standard model for the jet bulk motion (see, e.g., Spada 1999), the jet outflow velocity $v_{z}$ is given by

$v_{z} \simeq c\left(1-1 / \gamma_{\mathrm{L}}^{2}\right)^{1 / 2}$

and $i$ is the inclination angle between the jet axis and the line of sight. In the case of blazars, it is usually assumed that $i \simeq 1 / \gamma_{\mathrm{L}}$ (Robson 1996; Urry \& Padovani 1995). However, in the case of radio galaxies this assumption may not always hold and $i$ (or at least an upper limit) can be estimated by direct observations. In the following we develop the necessary formalism in general (without assumptions for the inclination angle) and then we will apply it to the radio galaxy $3 \mathrm{C} 66 \mathrm{~B}$ by assuming either $i \simeq 1 / \gamma_{\mathrm{L}}$ or the available radio observations which restrict the parameter range (Hardcastle et al. 1996).

For a typical blazar in which $i \simeq 1 / \gamma_{\mathrm{L}}$, we show in Fig. 2 the dependence of the expected light curve periodicity on $\gamma_{\mathrm{L}}$.

Thus, for the $3 \mathrm{C} 66 \mathrm{~B}$ galaxy it is expected that in the $\mathrm{X}$-ray and/or $\gamma$-ray band the signal periodicity $P_{\text {obs }}$ corresponding to $P_{\mathrm{K}} \simeq 1.05 \mathrm{yr}$ is in the range $\sim 10-150$ days as a function of $\gamma_{\mathrm{L}}$ in the case of $i \simeq 1 / \gamma_{\mathrm{L}}$. 3C 66B is a large source (several arcminutes in the direction of largest angular size) and is characterized by a jet and a counterjet with a high degree of asymmetry in the surface brightness up to distances of a few kpc from the radio nucleus, becoming more symmetric on largest scales.

The flux asymmetry may be explained by considering relativistic beaming, i.e. the whole jet material moves at the same bulk velocity $\beta \mathrm{c}$ and the jet and counterjet make the same angle $i$ to the line of sight. In this case, the emission is Doppler-beamed towards or away from the observer and the 


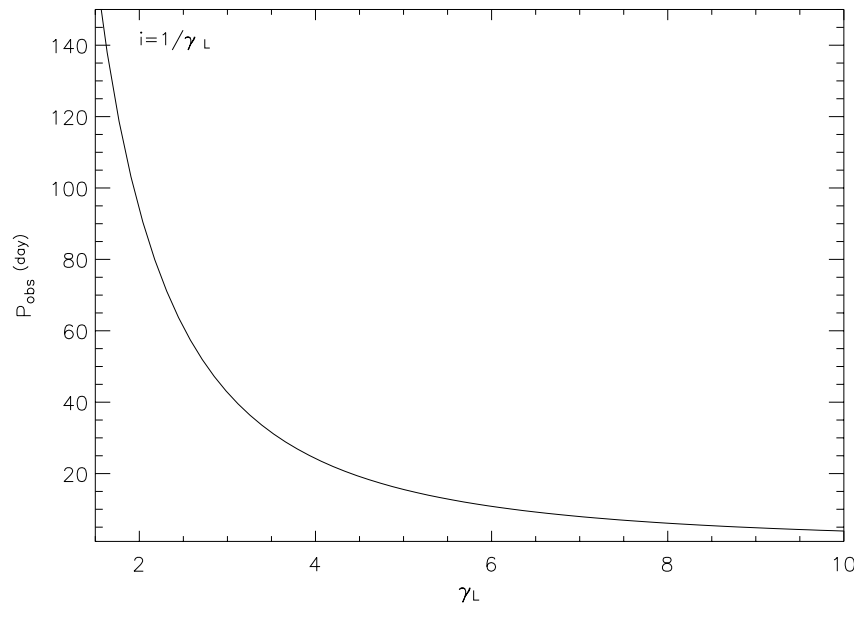

Fig. 2. The expected light curve periodicity as a function of the jet Lorentz factor $\gamma_{\mathrm{L}}$ is shown assuming the relation $i \simeq 1 / \gamma_{\mathrm{L}}$.

ratio $R$ between the jet and counterjet radio emission is given by (Hardcastle et al. 1996)

$R=\left(\frac{1+\beta \cos i}{1-\beta \cos i}\right)^{2+\alpha_{\mathrm{r}}}$,

where $\alpha_{\mathrm{r}}$ is the spectral index in the radio band. Measuring the integrated flux ratios of the jet and counterjet at different angular distances, it is possible to find $\beta \cos i$ as a function of distance from the nucleus. This procedure constrains $\beta \cos i$ to be in the range $0.25-0.6$. Moreover, values of $\beta \cos i$ around 0.6 constrain the angle to the line of sight of the emerging jet to be $i \lesssim 53^{\circ}$. For our purposes, leaving the jet inclination angle $i$ as a free parameter for the bulk motion velocity $\beta$ one has the obvious condition $\beta_{\min }<\beta<\beta_{\max }$, with $\beta_{\min }=0.25 / \cos i$ and $\beta_{\max }=0.6 / \cos i$. Consequently, the Lorentz factor is in the range $\left(1-\beta_{\min }^{2}\right)^{-0.5}<\gamma_{\mathrm{L}}<\left(1-\beta_{\max }^{2}\right)^{-0.5}$.

In this way we can evaluate the expected X-ray and/or $\gamma$-ray light curve periodicity as a function of $\gamma_{\mathrm{L}}$ for different jet inclination angles $i$ by using Eq. (4). In Fig. 3, we plot $P_{\text {obs }}$ for the angles $i=20^{\circ}, 30^{\circ}, 45^{\circ}$ and $50^{\circ}$. Note that the observed periodicity is always less than one year.

Let us further assume that the jet is emitted by the less massive black hole (of mass $m$ ) and that, as usual, the jet axis is parallel to the orbital angular momentum vector ${ }^{2}$. Moreover, we assume that the non-thermal X-ray and/or $\gamma$-ray radiation propagates outwards from the core along the jet with Lorentz factor $\gamma_{\mathrm{L}}$. In this way, the observed flux modulation due to Doppler boosting can be written as (Rieger \& Mannheim 2000)

$S(v)=\delta^{3+\alpha} S^{\prime}(v)$,

2 We would like to mention that a generalization of our treatment to the more general case with arbitrary direction of the jet axis with respect to the orbital angular momentum vector requires the introduction of a set of quantities (the position angles of the jet in the sky plane, the jet precession opening half-angle, the inclination angle of the orbital plane, etc.) following, for example, a similar approach to that proposed by Abraham \& Carrara (1998). However, this approach requires the knowledge of a number of parameters that are only weakly constrained by the observations. A comprehensive treatment of this case will be presented elsewhere.

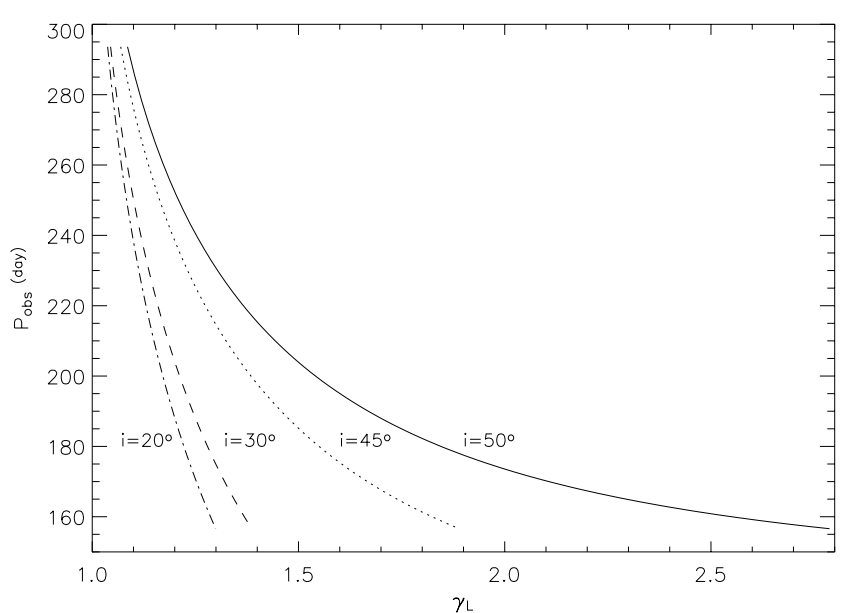

Fig. 3. The expected light curve periodicity as a function of $\gamma_{\mathrm{L}}$ is shown for different jet viewing angles $i$.

where $\alpha$ is the source spectral index ${ }^{3}$ and the Doppler factor is given by

$\delta=\frac{\sqrt{1-\left(v_{z}^{2}+v_{\mathrm{ls}}(\theta)^{2}\right) / c^{2}}}{1-\left(v_{z} \cos i+v_{\mathrm{ls}}(\theta) \sin i\right) / c}$.

Here, $v_{\mathrm{ls}}$ is the component of the less massive black hole velocity along the line of sight and $\theta$ the polar angle defining the position of $m$ in its orbit.

As discussed in De Paolis et al. (2002), the maximum and minimum values of the velocity $v_{\mathrm{ls}}$ are attained for $\theta=\pi / 2$ and $\theta=3 \pi / 2$, respectively, corresponding, through Eq. (8), to the two possible values $\delta_{\max }$ and $\delta_{\min }$ of the Doppler factor. With the assumption that the periodicity in the observed signal is due to the orbital motion of the binary black hole, one obtains from Eq. (7) the condition

$\delta_{\max } / \delta_{\min } \simeq f^{1 /(3+\alpha)}$,

where $f$ is the observed maximum to minimum flux ratio. Once the value of $v_{\mathrm{ls}}$ is known the previous equations give (for details see De Paolis et al. 2002)

$$
\begin{aligned}
\frac{M}{(m+M)^{2 / 3}}= & \frac{P_{\mathrm{obs}}^{1 / 3}}{[2 \pi(1+z) G]^{1 / 3}} \frac{c}{\sin i} \\
& \times \frac{f^{1 / 3+\alpha}-1}{f^{1 / 3+\alpha}+1}\left(1-\frac{v_{z}}{c} \cos i\right)^{2 / 3}\left(1-e^{2}\right)^{1 / 2} .
\end{aligned}
$$

VLBI observations may be used to constrain the masses of the two black holes in $3 \mathrm{C} 66 \mathrm{~B}$ so that the previous equation can be solved with respect to $f$ giving

$f=\left[\frac{1+k}{1-k}\right]^{3+\alpha}$,

with

$\begin{aligned} k= & \frac{M}{(m+M)^{2 / 3}} \frac{[2 \pi(1+z) G]^{1 / 3}}{P_{\mathrm{obs}}^{1 / 3}} \frac{\sin i}{c} \\ & \times\left(1-\frac{v_{z}}{c} \cos i\right)^{-2 / 3}\left(1-e^{2}\right)^{-1 / 2} .\end{aligned}$

\footnotetext{
${ }^{3}$ In the following, typical values of the power law index $\alpha$ are assumed to be in the range 1-2 (for details see Guainazzi et al. 1999; and Boller et al. 2000).
} 


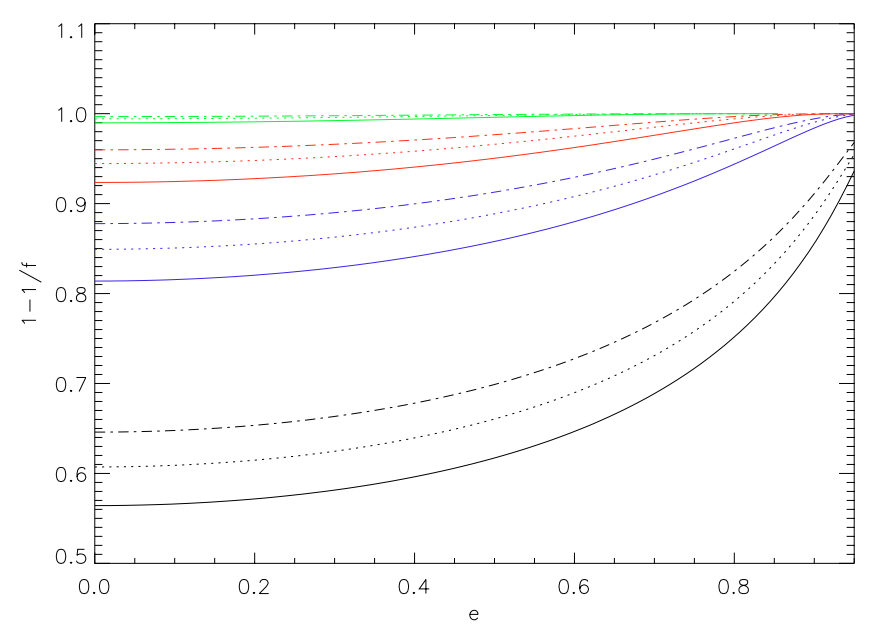

Fig. 4. The expected percentage flux variation of the X-ray and/or $\gamma$-ray light curves as a function of the orbit eccentricity. The curves have been obtained assuming, for the black hole maximum semi-major axis, $a \simeq 5.4 \times 10^{16}(1+q) / \eta \mathrm{cm}$ with $\eta \simeq 4$. Black, blue, red and green lines correspond to Lorentz factors $\gamma_{\mathrm{L}}$ of 2, 4, 6 and 10, respectively. Solid, dotted and dashed lines correspond to power law indices $\alpha=1,1.5$ and 2, respectively. Here we have also assumed $i \simeq 1 / \gamma_{\mathrm{L}}$. (This figure is available in color in electronic form at www. edpsciences.org)

The percentage flux variation given by the previous equation depends on the orbital parameters of the SMBHB systems, the jet viewing angle $i$ and the jet Lorentz factor $\gamma_{\mathrm{L}}$. As discussed above, for a typical blazar the latter quantities depend on each other (i.e. $i \simeq 1 / \gamma_{\mathrm{L}}$ ) while for radio galaxies like $3 \mathrm{C} 66 \mathrm{~B}$ this is not always true.

In Fig. 4 we show the expected percentage flux variation between the maximum and minimum signal as a function of the orbital eccentricity, for different values of both the Lorentz factor $\gamma_{\mathrm{L}}$ and the power law spectral index $\alpha$. Here we have considered the galaxy as a typical blazar, thus setting $i \simeq 1 / \gamma_{\mathrm{L}}$ in all the previous equations.

Since we expect that future radio observations at frequencies higher than $8.4 \mathrm{GHz}$ will improve the mapping of the SMBHB orbit in $3 \mathrm{C} 66 \mathrm{~B}$, we consider, as an example, the orbit semi-major axis to be $a \simeq 5.4 \times 10^{16}(1+q) / \eta \mathrm{cm}$, being $\eta \simeq 4$.

Note that in this case, using the orbit semi-major axis obtained by the $8.4 \mathrm{GHz}$ radio map (i.e. $a_{\max } \simeq 5.4 \times 10^{16}(1+$ q) $\mathrm{cm}$ ), Eq. (11) will be satisfied only for small Lorentz factor values $\gamma_{\mathrm{L}} \lesssim 2$. In this case, the expected flux ratio $f$ is high enough to make $1-1 / f$ approach unity for any value of the eccentricity $e$ (and therefore always imply $f \gtrsim 100$ ).

Let us now consider again the most realistic scenario according to which the jet inclination angle is $i \lesssim 53^{\circ}$ (Hardcastle et al. 1996). In this case, for each possible value of the inclination angle $i$ there exists a different set of allowed values of the Lorentz factor $\gamma_{\mathrm{L}}$ (see discussion above). Hence, using Eq. (11) we can evaluate the expected flux percentage variation for different jet inclination angles $i$. This is shown in Figs. 5a and 5b where we plot the expected percentage flux for two selected values of the jet inclination angle and $\gamma_{\mathrm{L}}\left(i=50^{\circ}\right.$ and $\gamma_{\mathrm{L}}=2$ in Fig. 5a, $i=30^{\circ}$ and $\gamma_{\mathrm{L}}=1.3$ in Fig. 5b).
As one can see, X-ray and/or $\gamma$-ray observations towards 3C 66B may make it possible to detect a possible periodic behavior in the light curves which can be directly used to fix the Lorentz factor $\gamma_{\mathrm{L}}$ (see Fig. 2). In addition, the detection of the signal flux ratio $f$ will allow to determine the orbit eccentricity $e$ of the SMBHB possibly hosted by the radio galaxy 3C 66B at least if the percentage flux variation is not close to unity, and also give information about the jet inclination angle $i$.

Note also that radio observations of the nuclear region of $3 \mathrm{C} 66 \mathrm{~B}$ have shown a strong flux variability (up to 35 per cent at high frequencies) on a time scale of months (Hardcastle et al. 1996) which might be due to the Doppler modulation effect of the jet and counterjet motion towards or away from the observer.

\section{Discussion and conclusions}

As stated in the previous section, if $3 \mathrm{C} 66 \mathrm{~B}$ really hosts an SMBHB in its center, a periodic behavior of the light curve in the X-ray and/or $\gamma$-ray bands and a certain flux ratio $f$ are expected. A measure of $P_{\text {obs }}$ and $f$ would allow getting both the jet Lorentz factor $\gamma_{\mathrm{L}}$ and the system orbit eccentricity $e$ through Figs. 2 and 4.

However, since the curves in Fig. 4 do not depend on the mass ratio $q$, also measuring $f$ does not make it possible to obtain a complete definition of the orbital parameters of the SMBHB. Thus, a third observation, i.e. the gravitational wave emission, is necessary to extract the value of $q$.

First, note that the emission of gravitational radiation makes the SMBHB orbit to shrink within the merging time-scale (Peters 1964; Fitchett 1987)

$T \simeq \frac{167 \tau c^{5}}{M m(M+m)} \int_{0}^{e_{0}} \frac{\left(1+\frac{121 e^{2}}{304}\right)^{1181 / 2299} e^{29 / 10}}{\left(1-e^{2}\right)^{3 / 2}} \mathrm{~d} e$,

where $\tau$ is a constant that depends on the initial orbit semi-major axis $a_{0}$ and eccentricity $e_{0}$.

In Fig. 6 the coalescing time scale $T$ for different values of the mass ratio $q$ (different lines) and two values of the semi-major axis $a$ (different colors) is shown as a function of the orbit eccentricity $e$. Models corresponding to the higher value of $a$ (red lines) are clearly unlikely since the corresponding coalescing time-scale is shorter than $10^{2} \mathrm{yr}$. On the contrary, models with the smaller semi-major axis (corresponding to that derived by using the $8.4 \mathrm{GHz}$ map) and $q \lesssim 10^{-3}$ always have coalescing time-scales longer than $\sim 10^{2}$ yr and may therefore be considered acceptable. Note also that, as previously stated, since future radio observations at frequencies higher than $8.4 \mathrm{GHz}$ will allow better constraint of the semimajor axis of the SMBHB system the true coalescing timescale of the massive black hole binary systems may be longer than that quoted here.

The SMBHB mass ratio $q$ may be determined if one could observe the gravitational wave spectrum. In Fig. 7 we present the expected gravitational wave spectrum for different values of the mass ratio $q$ and orbital eccentricity $e$. Straight lines correspond to the detection thresholds of the next generation of space-based interferometers LISA (Reinhard 2000) and ASTROD (Wu et al. 2000; Ni 2002). 


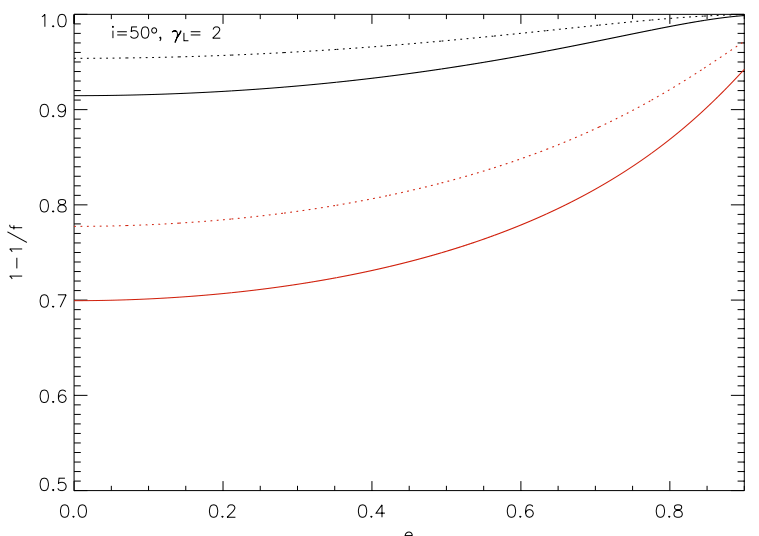

(a)

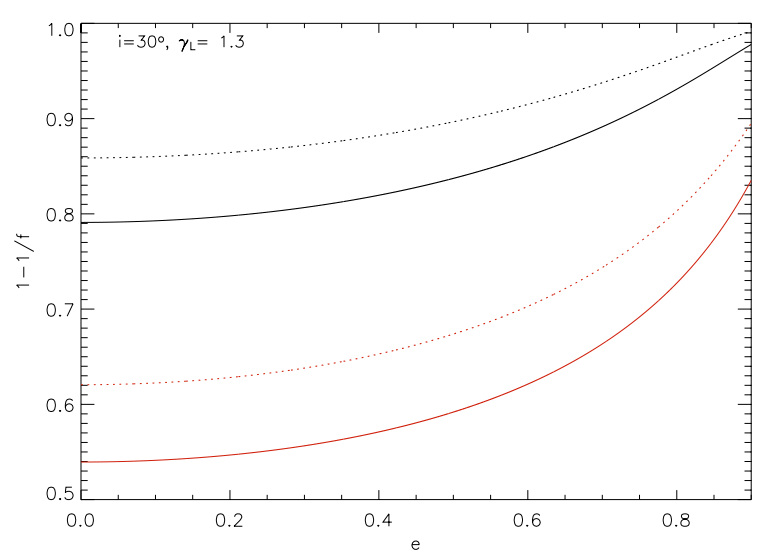

(b)

Fig. 5. The expected percentage flux variation of the X-ray and/or $\gamma$-ray light curves as a function of the orbit eccentricity. The curves were obtained assuming, for the black hole semi-major axis, $a \simeq 5.4 \times 10^{16}(1+q) / 2 \mathrm{~cm}$ (black lines) and $a \simeq 5.4 \times 10^{16}(1+q) / 4 \mathrm{~cm}(\mathrm{red}$ lines). Solid and dotted lines correspond to power law index $\alpha=1$ and 2, respectively. Here we are considering a more realistic geometry for the $3 \mathrm{C} 66 \mathrm{~B}$ galaxy jets. (This figure is available in color in electronic form at www. edpsciences.org)

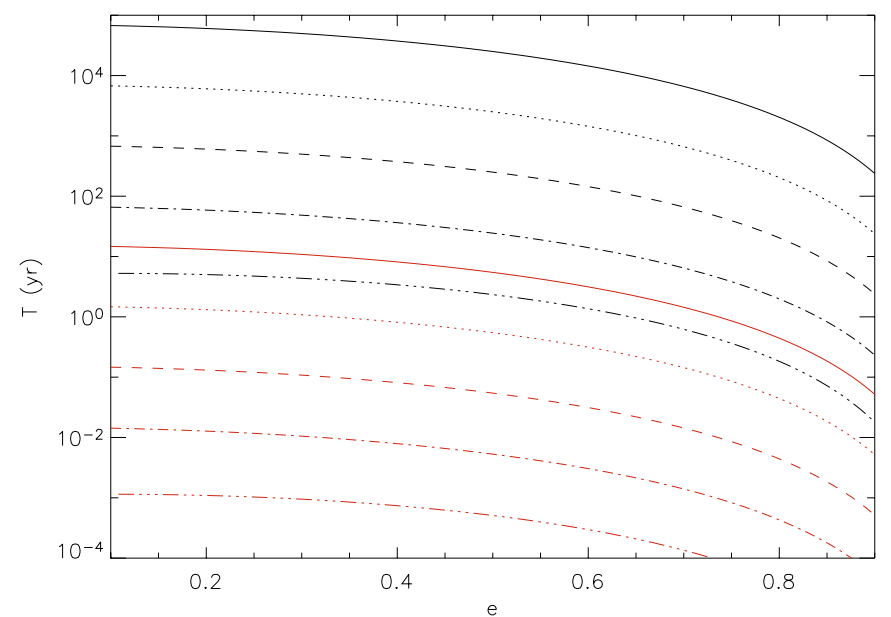

Fig. 6. The coalescing time scale $T$ as a function of the true orbit eccentricity $e$. Black and red lines correspond to the orbital maximum semi-major axis $a \simeq 5.4 \times 10^{16}(1+q) \mathrm{cm}$ and $a \simeq 2.9 \times 10^{17}(1+q) \mathrm{cm}$, respectively. Solid, dotted, dashed, dot-dashed and two-dot-dashed lines are for $q=10^{-5}, 10^{-4}, 10^{-3}, 10^{-2}, 10^{-1}$, respectively. (This figure is available in color in electronic form at www. edpsciences.org)

Thus, future X-ray and/or $\gamma$-ray observations may allow to obtain information about both the bulk motion of the jet $\left(\gamma_{\mathrm{L}}\right)$ and its precession and, consequently, confirm the SMBHB model for the radio galaxy $3 \mathrm{C} 66 \mathrm{~B}$. These measurements may also allow to define the semi-major axis $a$ and estimate the orbital eccentricity $e$ of the binary system as follows from Figs. 2 and 4. Moreover, the mass ratio $q$ may be attained from the observation of the emitted gravitational wave spectrum by using next generation space-based interferometers.

Note that recently a different method for constraining the properties of the SMBHB in 3C 66B has been proposed (Jenet et al. 2003). The main idea is to search for gravitational wave emission from the SMBHB system by using available timing data ( 7 yr up to now) from the radio pulsar PSR B1855+09

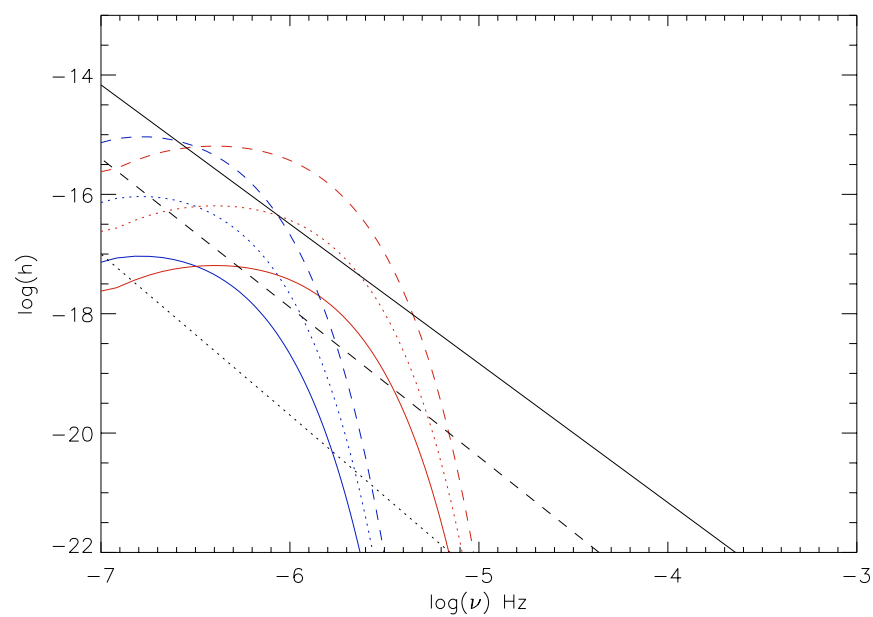

Fig. 7. The expected gravitational wave spectrum for the SMBHB in 3C 66B. Black, blue and red lines are for $q=10^{-5}, 10^{-4}, 10^{-3}$, respectively. Solid and dotted lines indicate eccentricity values of $e=0.7$ and $e=0.9$. Here we have assumed the semi-major axis to be $a \simeq 5.4 \times$ $10^{16}(1+q) \mathrm{cm}$. Solid, dashed and dotted straight lines show the planned sensitivity thresholds of LISA, ASTROD1 and ASTROD2 instruments. Here we have assumed an integration time of $5 \mathrm{yr}$. (This figure is available in color in electronic form at www. edpsciences.org)

(Kaspi et al. 1994). In fact, gravitational waves will induce periodic oscillations in the arrival times of the individual pulses of the pulsar. Numerical simulations performed for different parameters have shown that the proposed method can be used to solve the parameter degeneracy of the SMBHB in 3C 66B only for extremely small orbital eccentricity values $(e<0.03)$. In the other cases the method can not give a definitive answer using the current data (Jenet et al. 2003).

Acknowledgements. One of us (A.A.N.) would like to thank B. M. T. Maiolo for reading the manuscript and A. F. Zakharov for interesting discussion. We also would like to thank the anonymous referee for the suggestions. 


\section{References}

Abraham, Z., \& Carrara, E. A. 1998, ApJ, 496, 172

Begelman, M. C., Blandford, R. D., \& Rees, M. J. 1980, Nature, 287, 307

Boller, T., Keil, R., Trümper, J., et al. 2001, A\&A, 365, 134

De Paolis, F., Ingrosso, G., \& Nucita, A. A. 2002, A\&A, 388, 470

De Paolis, F., Ingrosso, G., Nucita, A. A., et al. 2003, A\&A, 410, 741

Fitchett, M. 1987, MNRAS, 224, 567

Guainazzi, M., Vacanti, G., Malizia, A., et al. 1999, A\&A, 342, 124

Hardcastle, M. J., Alexander, P., Pooley, G. G., et al. 1996, MNRAS, 278, 273

Kaspi, V. M., Taylor, J. H., \& Ryba, M. F. 1994, ApJ, 428, 713

Kormendy, J., \& Richstone, D. 1995, ARA\&A, 33, 581

Jenet, F. A., Lommen, A., Larsen, S. L., et al. 2004, ApJ, 606, 799

Leahy, J. P., \& Williams, A. G. 1984, MNRAS, 210, 929

Lehto, H. L., \& Valtonen, M. J. 1996, ApJ, 460, 207

Lobanov, P. 1998, A\&A, 330, 79

Matthews, T. A., Morgan, W. W., \& Schmidt, M. 1964, ApJ, 140, 35

Miller, H. R., \& McGimsey, B. Q. 1978, ApJ, 220, 19

Nakano, T., \& Makino, J. 1991, ApJ, 510, 166

Ni, W. T. 2002, Inter. Journ. Mod. Phys. D, 11, 947
Parma, P., Ekers, R. D., \& Fanti, R. 1985, A\&A, 59, 511

Peters, P. C. 1964, PR, 136, 1124

Pietila, H. 1998, ApJ, 508, 669

Rees, M. 1984, ARA\&A, 22, 471

Reinhard, R. 2000, LISA Detecting and Observing Gravitational Waves, in ESA Bulletin, 103, 33

Richstone, D., Ajhar, E. A., \& Bender, R. 1998, Nature, 395, 14

Rieger, F. M., \& Mannheim, K. 2000, A\&A, 359, 948

Robson, I. 1996, Active Galactic Nuclei (John Wiley and Sons)

Sadun, A., Sillanpaa, A., Takalo, L. O., et al. 1994, A\&AS, 185, 8807

Schoenmakers, A. P. 2000, MNRAS, 315, 371

Sillanpää, A., Haarala, S., Valtonen, M. J., et al. 1988, ApJ, 325, 628

Spada, M. 1999, Astropart. Phys., 11, 59

Sudou, H., Iguchi, S., Murata, Y., et al. 2003, Science, 300, 1263

Urry, C. M., \& Padovani, P. 1995, PASP, 107, 803

Wang, T. G., Zhou, H. Y., \& Dong, X. B. 2003, AJ, 126, 113

White, S. D. M. 1997, in The evolution of the Universe: Report of the Dahlem Workshop, ed. G. Börner, \& S. Gottlöber (Berlin), 227

Wu, A. N., Xu, X. H., \& Ni, W. T. 2000, Inter. Journ. Mod. Phys. D, 9, 201

Yu, Q. 2002, MNRAS, 331, 805 